\title{
OPTIMISING STRATEGIC DECISION MAKING IN WATER NETWORKS
}

\author{
C. Chiu $^{l^{*}}$, B. Jankovic-Nisic ${ }^{1}$, L. Pocock ${ }^{2}$, L. Murphy ${ }^{2}$, R. Alkhatib ${ }^{1}$, J. Cantone ${ }^{2}$, O. EriOlu ${ }^{1}$, J. Downs ${ }^{3}$ \\ ${ }^{1}$ Arcadis UK Engineering Water and Energy, 34 York Way N1 9AB, London, United Kingdom \\ ${ }^{2}$ Optimatics, 318 Adams Street 18th Floor 60606, Chicago, United States \\ ${ }^{3}$ Southern Water Services Planning and Resilience, Southern House Lewes Road, Falmer United Kingdom \\ * Corresponding author
}

\begin{abstract}
This paper presents a pilot project which developed a novel methodology aimed at increasing efficiency of the strategic planning process for water supply networks. Planning and investment processes are generally manual and iterative exercises. The pilot investigated if more advanced optimisation techniques could be used to stretch planning decisions by systematically incorporating operational decisions in the strategic planning process, considering business risks associated with various asset decisions, whilst improving the resilience of the network and minimising cost. The methodology was applied on a water supply zone within Southern Water Services and enabled Arcadis and Optimatics, in partnership with the water utility, to directly address the challenges set by The Water Services Regulation Authority in the UK (Ofwat) for the 2020-2025 regulatory period and beyond by innovating the strategic planning process. The project deployed a decision-making software, Optimizer, which uses metaheuristic algorithms to simulate hundreds of thousands of future water asset investment scenarios. Optimizer produces a pareto front of the most efficient planning options given user defined parameters of cost, water network performance and impact of sites outage on customers, i.e. resilience and business risk. This novel approach proved to enable a robust, traceable and thorough decision-making process to produce a set of business defined balanced asset plans.
\end{abstract}

\section{Introduction}

Planning and investment processes of water supply networks are generally manual and iterative exercises experienced by utility companies. This paper outlines a pilot project which employed a novel methodology as a means to increase the efficiency of strategic planning of water supply networks. The pilot challenged if more advanced optimisation techniques could be used to stretch planning decisions by systematically incorporating operational decisions in the strategic planning process, considering business risks associated with various asset decisions, whilst improving the resilience of the network and minimising cost.

The methodology was applied on a water supply zone within the Southern Water Services and enabled Arcadis and Optimatics, in partnership with the water utility, to directly address the challenges set by The Water Services Regulation Authority in the UK (Ofwat) for the 2020-2025 regulatory period and beyond by innovating the strategic planning process. This project considered all four themes set by OFWAT: affordable bills, resilience, innovation and great customer service (Ofwat, 2017; Optimatics, 2018).

The pilot project deployed a decision-making software, Optimizer, which uses metaheuristic algorithms (Optimatics, 2014) to simulate hundreds of thousands of future water asset investment scenarios. Optimizer provides a pareto front of the most efficient planning options given user defined parameters of cost, water network performance, impact of asset outage on customers i.e. resilience, and business risk.
The challenge is complex - assessing numerous new capital asset solutions including water treatment plants, additional pipelines, pumping stations and reservoirs to improve resilience. These capital asset solutions are considered as sites. Sites consist of a cohort of assets performing an operational function e.g. a water treatment plant's associated infrastructure is considered as one site.

The sites built into a hydraulic model, modelling water quality and hydraulic performance, while satisfying Southern Water standards and improving customer levels of service. Bespoke controls and scripts were written to assess the resilience by identifying the number of properties that would lose water supply if sites were out of service.

The approach proved to enable a robust, traceable and thorough decision-making process to answer question such as:

- What does a 'balanced' asset plan look like?

- What is the business risk exposure for a given asset plan?

- What is the sensitivity of the plan to changing parameters e.g. a future increase in water demand or environmental pollutant risks?

- What is the cost of improving resilience for a given site failure? 


\section{Background}

\subsection{Optimisation of water supply networks}

There has been extensive research undertaken on water network optimisation as outlined by Mala-Jetmarova et al. 2018, which alone reviewed just under 300 papers. The findings revealed scores of optimisation techniques have been studied, however most studies limited the number of objectives to one or at most two.

In real world problems, there are numerous decision variables which range from hydraulic, environmental, economic to business risks. Different parts of the utility business often have differing priorities; a multi-objective optimisation is essential to reflect real world conditions. Multi-objective methods more reflect real business operations, which offers a set of solutions (Mala-Jetmarova et al., 2018) from which the business can further evaluate trade-offs of parameters. Additionally, often there are numerous decision variables resulting with possible hydraulic solutions in the order of the billions - even experienced engineers may not converge upon the most 'optimal' solutions (Simpson et al., 1993).

Of the numerous optimisation methodologies proposed computing power has been a limiting constraint; only in the 20th century has there been major steps forward in our abilities to implement advanced analytical methods (Savić et al., 2018).

\subsection{Regulatory context}

For the upcoming regulatory period, Ofwat has set four themes: affordable bills, resilience, innovation and great customer service (Ofwat, 2017). To meet the challenge of the regulator, this project addresses all four themes. Costs and benefits of infrastructure and levels of service to customers have always been a primary focus to Southern Water, however, resilience is now to be explicitly addressed and embedded within business plans.

Resilience is a central tenant of the AMP 7 regulatory period, due to the new statutory duty placed on it by the UK government (Water Act 2014, Cabinet Office, 2017). Ofwat defines resilience as "ability to cope with, and recover from, disruption, and anticipate trends and variability in order to maintain services for people and protect the natural environment now and in the future" (Ofwat, 2017). In Ofwat's PR19 methodology, it requested all water companies to place customers at the heart of their resilience thinking, consider affordability and impacts of their investment choices on bills (Ofwat, 2017).

\subsection{Enhancing the Resilience Methodology}

This paper outlines a complementary methodology to an Arcadis developed Resilience Methodology which measures resilience. As an enhancement to the current resilience assessment developed by Arcadis with Southern Water, this pilot also aimed to explore how Southern Water could further model resilience and incorporate the findings in a strategic investment plan by integrating hydraulic models into the existing methodology.
The consequence-led resilience tool which focuses on service continuity and is based on a definition of resilience aligned to Ofwat's thinking, and subsequently endorsed in Ofwat's PR 19 consultation paper (Ofwat, 2017). It puts customers at the heart of investment decisions and enables consideration of various interventions beyond capital expenditure; the tool allows utilities to be able to pin point where and how operations and maintenance activities contribute to resilience. The approach measures the ability of Southern Water to respond to external shocks for a water supply zone including flooding, critical asset failure, contamination, raw water loss, malicious damage and site communication failures.

The risk calculation reflects this adequately by building on the international $\mathrm{J} 100$ resilience framework developed by the US Department of Homeland Security and the UK Cabinet office 4R's (Redundancy, Response and Recovery, Resistance, and Reliability) (Cabinet, 2017) as outlined in Equations (1) and (2).

Risk $=C * V * T$

Resilience $=$ Risk $* C F$

Equation (1) shows that risk is dependent on $\mathrm{C}$ (consequence), V (vulnerability) and $\mathrm{T}$ (threat likelihood). While Resilience is the dependent on risk, defined in Equation (1) and a control factor (CF), which is calculated using the UK Cabinets 4Rs. Figure 1 shows how risk scores relate to each of the $4 \mathrm{R}$ control factors.

\section{Figure 1 Resilience Score Assessment}

\section{RESILIENCE SCORE ASSESSMENT}

RISK SCORE CONTROL FACTOR

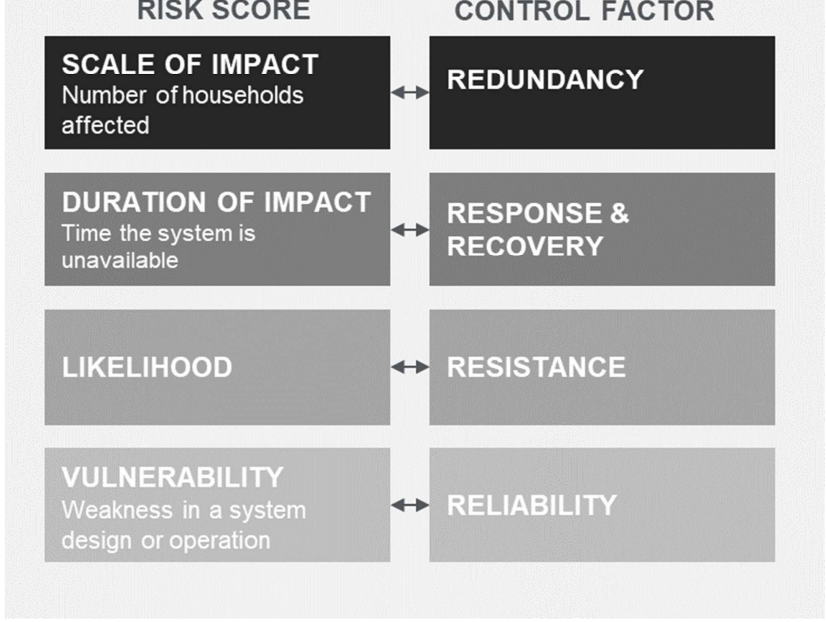

A natural progression is to assess one of the Rs, Redundancy, using a hydraulic model. The same sites identified in this resilience tool were used to assess the scale of impact if one of the sites were to fail i.e. the number of customers on each demand node suffers a decreased level of service. 


\section{Methodology}

Typically, optimisation models have three main components: objectives, constraints and decision variables (MalaJetmarova et al., 2018). This methodology does not deviate from these basic components but has allowed the complexity of a multi-objective optimisation problem to be systematically built into the model.

The key steps of the methodology follows:

1. Business consultation to determine key business drivers, associated costs and capital and/or operational asset solutions

2. Set up objective functions

3. Formulation of capital and operations decision into the hydraulic model and Optimizer

4. Define design criteria and business risks

5. Define costs associated with decisions

6. Evaluate interim optimisation runs for possible further refinement and review best performing asset plans

7. Perform sensitivity analysis to check stability of solutions.

\subsection{Objective functions and key business drivers}

The aim of this pilot was to understand if there was a more efficient, transparent and holistic methodology which can be used to develop an optimised solution which will address the key business drivers simultaneously for a defined set of potential asset options and interventions.

The planning and investment process is often iterative and manual and spans across different arms of the business; defining the optimum level of investment, given the suite of interventions. The methodology aimed to provide decision makers with a tool to make robust and transparent decisions and to easily assess any trade-offs between cost and customer benefits.

An intensive consultation period is required to understand key business drivers, and the suite of options available to fulfil these objectives.

The three defined objectives are:

- Balancing total expenditure (TOTEX) of the combination of the capital and operational asset options,

- Resilience impact on properties interruptions due to assert failure, and

- Hydraulic performance, including water quality.

\subsection{Optimizer model development: design criteria and decisions}

This pilot deployed Optimizer, an outcomes driven analytics platform which uses artificial intelligence combined with a custom developed high performing computing stack that allows planners to leverage cloud computing and metaheuristic optimisation techniques for capital and operational infrastructure water resource problems. The algorithms utilised have been adapted from academic research (Gibson, 2006) and enhanced with heuristics developed through years of applying the algorithms to industrial scale problems.

Optimizer employs Computational Intelligence (CI) in the form of a range of different optimization algorithms to solve complex water engineering problems. The CI embedded in Optimizer is able to search hundreds of thousands of potential solutions and converge on least-cost, hydraulically-feasible solutions.

Typically, engineers use trial and error to plan system improvements or new developments for water systems. However, the number of combinations of system improvements can be greater than $10^{20}$, even for relatively small problems. The process of selecting system improvements and sizes, costing the improvements and evaluating the system hydraulics is automated using Optimizer. CI then drives the search for even better solutions.

At the time of developing this methodology, the Optimizer platform used an EPANET hydraulic model. As this was an assessment for a future strategic plan, in this case, the base model was future water stressed demand scenario to the year 2030 and reflected a typical day of operations.

The decision variables are potential capital asset and operational solutions developed during a consultation period with stakeholders. The formulation of these options requires inputs from across the business and teams who have historical knowledge of the network and would be operating the network in the future. Each of these potential solutions are built into the hydraulic model which is then assessed by Optimizer, along with their combinations and permutations. Workshops are required to develop and refine the capital and operating alternatives for the network. Additionally, constraints such as capital and operational costs need to be gathered for each of the decision variables. A common view of cost elements is possible across business teams and can be built with user defined level of cost granularity.

As with all hydraulic designs, design criteria are defined. In this case, conforming to Southern Water's design standards for hydraulic performance e.g. minimum pressures, velocities.

\subsection{Incorporating business risks into an optimisation}

In order to measure the effectiveness of a design against numerous parameters, a scoring system is required to evaluate each asset solution. Business risk can be systematically built into the Optimizer model when the design criteria is violated.

Penalties are assigned proportionally to simulation runs where design criteria are outside of the pre-defined boundaries, differentiating them from the solutions where the design criteria have been met and thus ranking simulations with their hydraulic performance. This method means that instead of building in design criteria as constraints, they are treated as objectives. The level of which these objectives are not fulfilled can reflect the business's exposure to risk, which can 
simulate real world consequences such as fines or violations of Outcome Delivery Incentives (ODI).

Optimizer has been designed to minimise the cost of asset plans; penalty functions can be set to find the lowest economic cost i.e. lowest financial cost to the business, environmental cost, and impact of customer's level of service.

\subsection{Simulations and evaluation}

Due to the multi-objective nature of this methodology, there will be trade-offs between the objectives; an output of the Optimizer model will be a pareto set which the business is able to further assess, evaluate and refine.

Understanding the trade-offs, helps decision makers assess the level of risk they are paying or forgoing for each asset plan.

\section{Use case: A Southern Water strategic supply network}

A water supply network in Southern Water was selected as a case study. The supply area has a strategic network length of $118 \mathrm{~km}, 13$ reservoirs, 11 ground water sources and serves 96,000 properties, just under $5 \%$ of all Southern Water's water supply customers.

After a consultation period with Southern Water, the specific key business drivers for this supply zone to be addressed were to:

- Improve system redundancy (as a component of resilience),

- Address rising groundwater nitrate concentration risks prior to exceeding PCV values $(11.2 \mathrm{Nmg} / \mathrm{l})$, and

- Address rising costs of nitrate removal in the most cost/effective way.

Additionally, the consultation period also provided the capital site and operational solutions and their associated costs. These, the decision criteria, fell into five site categories:

- One increased import of treated water from a neighbouring water supply zone,

- 23 duplicate trunk mains,

- Increase capacity of one reservoir,

- Four new raw water cross connections, and

- Rationalise ten nitrate sites.

The above business drivers were built into the three objectives of the optimisation model:

- $\quad$ Minimise TOTEX - Capital and operational sites.

- Zonal Resilience - A function of the number of properties at risk of pressures being less than existing pressures during an asset outage.

- Hydraulic performance - As defined in the design criteria.

The design criteria defined consisted of:
- Water quality - This was set to a conservative $10 \mathrm{mg} / \mathrm{L}$

- Network Pressure - Network nodes were set to minimum pressure operational standards, and demand nodes were defined as current minimum pressure.

- Velocity - Set to a defined maximum.

- Treatment works capacity - These were set to the maximum allowable volume, Peak Deployable Output (PDO), at the raw water sources.

Penalty violations were also defined alongside design criteria to incorporate business risk. The key penalty violations assigned were:

- Water Quality - The largest penalties were assigned to violations of nitrate concentrations of more than $10 \mathrm{mgN} / \mathrm{L}$ as water quality safety to customers always should be prioritised.

- Pressures - Penalties to violations to level of service customers were weighted to the number of customers on each demand node.

- Importing water - A cost saving was assigned if the volume imported was lower than current levels to encourage network to decrease reliance on water imports.

- Volume extracted - If the volume extracted from sources exceeded the PDO, the level of penalties reflected a realistic fine that Southern Water could incur.

Due to the specific business drivers this pilot required, bespoke scripts were built into each Optimizer simulation run namely for water quality assessment and to calculate a resilience score for each simulation, as seen in Figure 2.

Figure 2 Optimizer model simulation runs

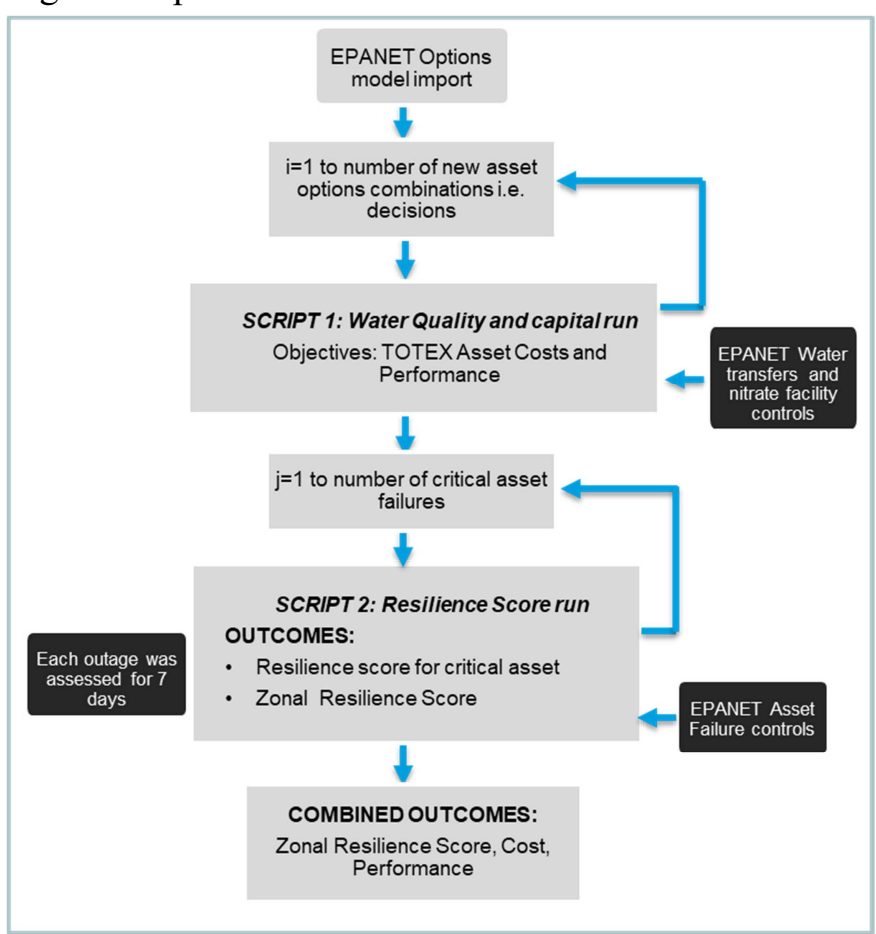




\subsection{Challenges \\ 4.1.1 Technical challenges}

The resilience script has been built on the foundation of the resilience assessment methodology, described in section 2.3, already completed for this supply zone.

A 'Resilience Factor', which is the product of the three of the four Rs (likelihood, vulnerability, resilience control factors) (see Figure 1), was applied each site to be assessed. A bespoke script calculated the fourth R, redundancy, by creating an outage scenario of each of these sites. These sites corresponded to the same sites which were assessed in the previous resilience methodology, which included 17 sites (transfer mains, booster stations and reservoirs) for consistency. As demonstrated in Figure 2, the redundancy assessment was designed to be carried out all within the same simulation.

Additionally, special attention has been given to building EPANET hydraulic model workarounds for nitrate treatment works as a facility and their extensive accompanying controls.

Although there is no limit to the number of different sites that can be considered in each optimisation run, the complexity of setting up the network for every run required numerous controls to be built to achieve hydraulic stability. Focusing on one type of site or limited number of sites would make setting up of the controls more manageable.

\subsubsection{Challenges in optimisation model formulation}

When formulating the optimisation model, defining the inputs were complex, especially when considering the appropriate granularity for a strategic approach.

Optimizer allows a common view of cost elements and allows user defined level of granularity, which would differ in importance to different parts of the business. For example, solution engineers may only be interested in relative costs while strategic planners may require more detailed costs for business plans.

Additionally, defining the level of granularity of asset groupings was also difficult. Sites can be further broken down into smaller cohorts to address different level of risk associated with, for example, critical crossings or different cost of failure at critical locations.

It was also noted that increasing the design criteria, options and cost granularity contributes to the computing power required for Optimizer to converge on a solution.

\section{Findings and next steps}

\subsection{Results}

As seen on Figure 3, the boundary of the pareto set represents the most efficient asset plans; for best hydraulic performance that can be achieved for a given TOTEX. The pareto set was compared to a manually derived plan (which did not consider cost, resilience and performance within one optimisation model). As seen in Figure 3, a manually derived plan does not sit on the pareto front. For an equivalent performance of the manually derived plan, this model shows this can be achieved for approximately $30 \%$ cost saving. Additionally, for an equivalent asset TOTEX, it also shows that a significant improvement in hydraulic performance.

\section{Figure 3 Optimizer output - pareto set of asset plans}

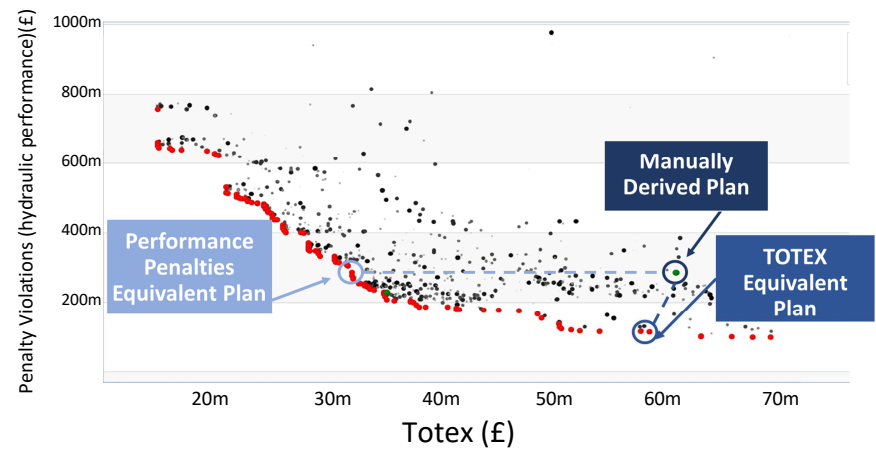

The pareto curve gives insight to the trade-off between hydraulic performance and asset TOTEX, the flattening shows the diminishing returns of further investment.

Due to the instability of the hydraulic model, the resilience scores were evaluated from selected asset plans on the pareto set, as part of the review of the best performing asset plans. Although not undertaken within the same simulation, Figure 4 shows distinct groups of asset plans and their cost of comparative resilience. Inspection of these step changes, resilience was grouped as: (A) asset plans with a relative poorer resilience score against other asset plans, (B) moderate resilience score and $(\mathrm{C})$ the highest achieved resilience score for a higher TOTEX.

\section{Figure 4 Cost of Resilience}

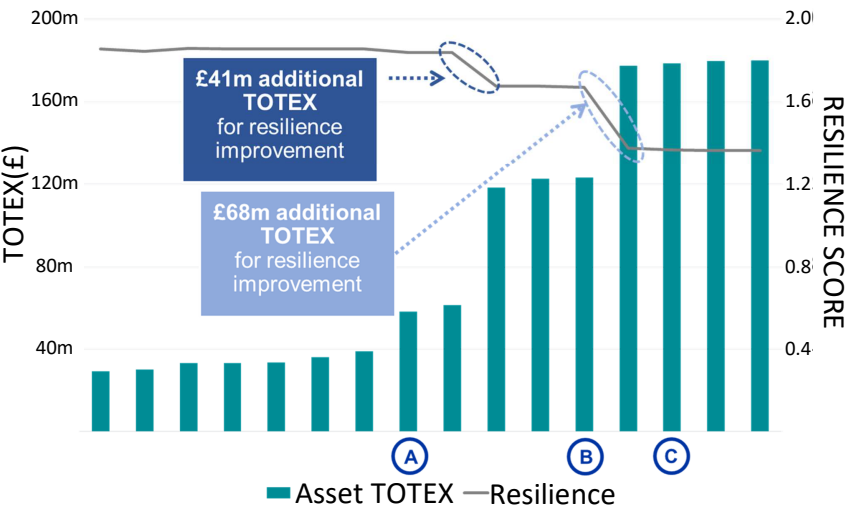

\subsection{Lessons learned and next steps}

\subsubsection{Hydraulic model}

This pilot was reliant on the conversion of the existing model from Infoworks WS Pro to EPANET. The viability of the integration of Optimizer into the business could be contingent on the ease of using existing hydraulic models, in Southern Water's case is Infoworks WS Pro, avoiding the conversion into EPANET. 
As outlined above, the effort building in the capital and operational models was significant due to fail-safe controls required to ensure model stability in the EPANET model. Using more sophisticated hydraulic modelling software on the market could increase the likelihood of hydraulic model stability. Although, it must be noted that the complexity stemming from evaluation of multiple system options is likely to be required regardless of the hydraulic software deployed.

\subsubsection{Optimisation model refinement and sensitivity analysis}

After the first Optimizer's pareto set was produced, the optimisation model formulation required further refinement to gain further insight into the parameter trade-offs. This iteration is less about selecting appropriate technical asset options, but more to understand sensitivity of business risk trade-offs and hydraulic performance for different levels of investment.

Further sensitivity assessment and refining penalty functions would improve the way the business drivers are optimized including ODIs. For example, it was apparent the weighting of penalty violations for the level of service to customers were too high compared to water quality violations and were easily adjusted.

Next steps would consider feasibility of asset plans to arrive to a more realistic set of notional solutions which can be further value engineered. The convergence of the best set of solutions, formulated balanced asset plans, can be achieved relatively rapidly giving more emphasis on business decisions and value engineering.

\subsubsection{Benefits and further use cases}

A methodology has been defined by working through this pilot use case, however a clear business processes needs to be derived. Clear mapping of processes applied to each business case use will be a key component to ensure visibility and consistency. A benefit of this methodology is the transparency of costs and business risks, which was apparent when the use case optimisation model was amended by different project team members with minimal effort. Business risk impacts can be hydraulically modelled i.e. ODIs, fines providing further insight into impacts of new capital assets or operation of assets. This ensures transparency and traceability in the solution development.

Transparency of Southern Water's solution engineering can be further enhanced by relying on processes and procedures by systematically incorporating the knowledge of those who have an in-depth knowledge of the water network. Additionally, although Optimizer evaluates options, engineering judgement of Optimizer results are still required to ensure designs meet business standards as more than one suitable option can be found.

The flexibility of Optimizer can allow for different use cases; the penalties can be included, excluded or adjusted to fit different models. For example, strategic business decisions can be reflected in the penalties with realistic fines for performance failures. Meanwhile, for hydraulic engineering designs, hydraulic performance penalties can be relative rather than absolute, while asset TOTEX costs are reflective of the supply chain costs.

Additionally, apart from standard set of parameters, the methodology can incorporate external asset, performance and environmental data in an extended property. This enables it to be used for a variety of custom-built applications, e.g. in this case resilience and water quality.

Southern Water can take most advantage of this methodology if there is an understanding of what key inputs Strategy, Planning and Resilience, and Operations can contribute to the optimisation model, as the granularity of understanding of various business risks differ across the business.

\section{Conclusions}

This multi-objective methodology has evaluated combinations and permutations of dozens of capital and operational asset solutions to improve network performance, cost efficiency and resilience of a supply network. This use case has shown that compared with a manually derived plan (which did not consider cost, performance and resilience in one optimisation model), for the same cost there was approximately between a $20-50 \%$ improvement in hydraulic performance, and a cost improvement of approximately $30 \%$ for an equivalent hydraulic performance. The convergence to a set of 'balanced' asset plans was achieved rapidly and efficiently.

This methodology enables a transparent and traceable strategic planning process while systematically incorporating business risks into the same assessment. The output, a pareto set, can be analysed further in detail and understood in the context of the business appetite for risk against ODIs and set budgets.

The success of this methodology is contingent on the business adopting this approach, due to the multi-disciplinary stakeholders required. The exercise is complex and requires the input of costing teams, engineering, planning and operations. Extensive consultation is required in order to build an optimisation model which reflects risks across the whole business.

By using a wholistic approach to strategic asset planning, we were able to address complexities of incorporating multiple parameters to achieve balanced strategic asset plans within one assessment.

\section{References}

Cabinet Office (2017) Public Summary of Sector Security and Resilience Plans, London, United Kingdom

Gibson C (2006), https://www.adelaide.edu.au/lumen/issues /11823/news11842.html (accessed 13th January 2019) The University of Adelaide Magazine: Lumen - Engineered for Success 
Mala-Jetmarova H et al. (2018) Lost in Optimisation of Water Distribution Systems? A Literature Review of System

Design. Water, 10(3), 307, MDPI AG

http://dx.doi.org/10.3390/w10030307

Ofwat (2017) https://www.Ofwat.gov.uk/wp-

content/uploads/2017/12/Final-methodology-1.pdf Delivering Water 2020: Our final methodology for the 2019 price review (accessed 12th January 2019)

Optimatics (2014) http://optimatics.com/optimatics-positionsitself-for-future-growth/ (accessed 14th September 2018) Optimatics Raises Capital to Accelerate Growth

Optimatics (2018) http://optimatics.com/southern-water-andarcadis (accessed 14th September 2018) Optimatics ${ }^{\mathrm{TM}}$ platform selected by Southern Water and ARCADIS for water transmission blending analyses

Savić et al (2018) History of Optimization in Water Distribution System Analysis. 1st International WDSA / CCWI 2018 Joint Conference, Kingston, Ontario, Canada

Simpson A et.al (1993) Pipe network optimisation using genetic algorithms. Specialty Conference of Water Resources Planning and Management Division (Katherine Hon (ed)), American Society of Civil Engineers, Seattle, Washington, USA, May, pp392-395.

Water Act 2014, c.22

http://www.legislation.gov.uk/ukpga/2014/21/section/22/enac ted (accessed 7th January 2019) 